\title{
First Insights on the Diversity of Edible Grasshoppers in South Africa by DNA Barcoding of Ethnospecies ${ }^{\dagger}$
}

\author{
Megan Hendrickse ${ }^{1}$, Bronwyn Egan ${ }^{2}$ and Barbara van Asch ${ }^{1, *}$ \\ 1 Department of Genetics, Stellenbosch University, Private Bag X1, Matieland 7602, South Africa; me- \\ gandhendrickse@gmail.com; bva@sun.ac.za \\ 2 Department of Biodiversity, University of Limpopo, Private Bag X1106, Mankweng 0727, South Africa; bron- \\ wyn.egan@ul.ac.za \\ * Correspondence: bva@sun.ac.za \\ † Presented at the 1st International Electronic Conference on Entomology (IECE 2021), 1-15 July 2021; \\ Available online: https://iece.sciforum.net/.
}

\begin{abstract}
Orthoptera are widely consumed in South Africa, but the actual diversity of species is largely undocumented. We used DNA barcoding to catalog seven ethnospecies of edible grasshoppers (Malefiswane, Tatakgope, Makahlodi, Mamaroping, Makihla, Makwitla, Tlatlawele) and edible Vlei grasshoppers. Genetic clustering and genetic divergences allowed for the identification of 14 genetic groups in Acrididae and one in Pyrgomorphidae. Species identification by sequence queries against BOLD Systems and GenBank was inconclusive in most cases. We confirm that DNA barcoding in Orthoptera is challenging due to NUMTs. Species diversity of edible grasshoppers in South Africa is high, and some may be suitable for mass-rearing.
\end{abstract}

Keywords: COI; genetic groups; Orthoptera; NUMTs; species identification

Citation: Hendrickse, M.; Egan, B.; van Asch, B. First Insights on the Diversity of Edible Grasshoppers in South Africa by DNA Barcoding of Ethnospecies, in Proceedings of the 1st International Electronic Conference on Entomology, 1-15 July 2021, MDPI: Basel, Switzerland, doi:10.3390/IECE-10371

Published: 30 June 2021

Publisher's Note: MDPI stays neutral with regard to jurisdictional claims in published maps and institutional affiliations.

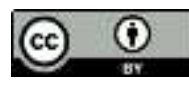

Copyright: (c) 2021 by the authors. Submitted for possible open access publication under the terms and conditions of the Creative Commons Attribution (CC BY) license (http://creativecommons.org/licenses /by/4.0/).

\section{Introduction}

Edible insects are widely consumed worldwide except in Western and Westernized societies. Insects generally have a high nutritional value and less environmental impact than conventional livestock and an advantageous feed conversion rate. The trade and consumption of edible insects from a variety of taxonomic groups contributes to the livelihoods and diet of many communities [1]. Orthoptera (grasshoppers, locusts and crickets) are primarily harvested from the wild in stark contrast with e.g., Thailand where cricket farming techniques were introduced 20 years ago and presently include over 20,000 small and large-scale enterprises that serve as the main source of income for many people [2]. Thus, commercial exploitation of indigenous Orthoptera as a food resource already consumed by many South Africans could assist in addressing food insecurity, elevate traditional food cultures and promote economic development [3]. This work aimed at compiling a reference catalogue of the ethnospecies of grasshoppers utilized in South Africa for future selection of candidate species for mass-rearing in insect farm environments.

\section{Materials and Methods}

\subsection{Sample Collection, DNA Extraction, PCR Amplification and Sequencing}

Ethnospecies of edible grasshoppers were identified and collected in the Limpopo Province of South Africa between February and April of 2020, with the assistance of local communities. DNA from individual specimens was extracted using a standard phenolchloroform protocol and barcoded by Sanger sequencing using a combination of univer- 
sal invertebrate primers and new taxon-specific primers. PCR amplifications were performed using the QIAGEN Multiplex PCR Kit (QIAGEN), according to manufacturer's protocol. Sanger sequencing was performed at the Central Analytical Facilities (CAF) of Stellenbosch University, South Africa. Sequences were manually inspected, edited and translated with Geneious Prime v2020.1.2. to detect potential premature stop codons and frameshift mutations.

\subsection{BLAST and BOLD Searches, Genetic Clustering and Genetic Divergences}

Species identification of the specimens was attempted by querying the new DNA barcodes against the BOLD Systems database (https://www.boldsystems.org/) and by using a BLASTn on NCBI database (www.ncbi.nlm.nih.gov). All available COI sequences for the relevant taxonomic groups were downloaded from GenBank (November 2020). Sequences $<500 \mathrm{bp}$, sequences with identical nucleotides, and those that did not overlap with the standard barcoding region were excluded from downstream analyses. Sequence alignments were performed with the MAFFT algorithm in Geneious Prime. Patterns of genetic clustering were recovered with Neighbour-joining (NJ) trees under the Kimura 2parameter (K2P) model in MEGA X. Genetic divergence was estimated as maximum pairwise distance (max p-distance, \%) in MEGA-X. Statistical support for NJ trees and p-distances was based on 1,000 bootstrap replicates.

\section{Results and Discussion}

\subsection{Challenges of DNA Barcoding for Species Identification in Orthoptera}

The objective of this work was to catalog the diversity of ethnospecies of edible grasshoppers in South Africa. Only $77 \%$ of the collected specimens (54/70) were successfully barcoded after PCR attempts with several primer combinations, and ambiguous base calls were evident in many sequences. The identification of genetic groups was not compromised but the hypothesis that Orthoptera genomes are rich in NUMTs and that therefore DNA barcoding in this group is particularly challenging, was supported [4-5].

\subsection{Edible Grasshoppers in South Africa fall into 15 Genetic Groups}

Sequence queries against BOLD Systems and GenBank allocated most specimens to six subfamilies in Acrididae, and to one species in Pyrgomorphidae. In most cases, the paucity of publicly available sequence data did not allow for inference of the species. Clustering analyses and estimates of genetic divergence recovered 15 genetic groups likely representing distinct species (max p-distances $<3 \%$ ) (Table 1; Figure 1).

Table 1. Ethnospecies of edible grasshoppers found in South Africa with genetic groups based on max p-distances and taxonomic identification based on BLASTn and BOLD System searches.

\begin{tabular}{lllll}
\hline Ethnospecies & Genetic group & Max p-distance (\%) & Family: Subfamily & Species \\
\hline Vlei Grasshopper 1 & A & 0.34 & Acrididae: Acridinae & Undetermined \\
Malefiswane & B1 & 1.37 & Acrididae: Acridinae & Undetermined \\
Malefiswane & B2 & 1.97 & Acrididae: Acridinae & Undetermined \\
Tatakgope & C1 & 0.31 & Acrididae: Cyrtacanthacridinae & Undetermined \\
Tatakgope & C2 & 0.63 & Acrididae: Cyrtacanthacridinae & Cyrtacanthacris sp. \\
Makahlodi & D & 1.46 & Acrididae: Eyprepocnemidinae & Undetermined \\
Mamaroping & E1 & 2.85 & Acrididae: Oedipodinae & Morphacris sp. \\
Makihla & E2 & 0.00 & Acrididae: Oedipodinae & Undetermined \\
Makwitla & E3 & 0.17 & Acrididae: Oedipodinae & Gastrimargus sp. \\
Makihla & E4 & 0.00 & Acrididae: Oedipodinae & Undetermined
\end{tabular}




\begin{tabular}{lllll} 
Makihla & E5 & 1.46 & Acrididae: Oedipodinae & Undetermined \\
Makihla & E6 & 0.00 & Acrididae: Oedipodinae & Undetermined \\
Vlei Grasshoppers 2 & E7 & 1.60 & Acrididae: Oedipodinae & Aiolopus sp. \\
Vlei Grasshoppers 3 & G & 3.25 & Acrididae: Spathosterninae & Spathosternum nigro- \\
Tlatlawele & F & 0.18 & Pyrgomorphidae: Pyrgomorphinae & Zonocerus elegans \\
\hline
\end{tabular}

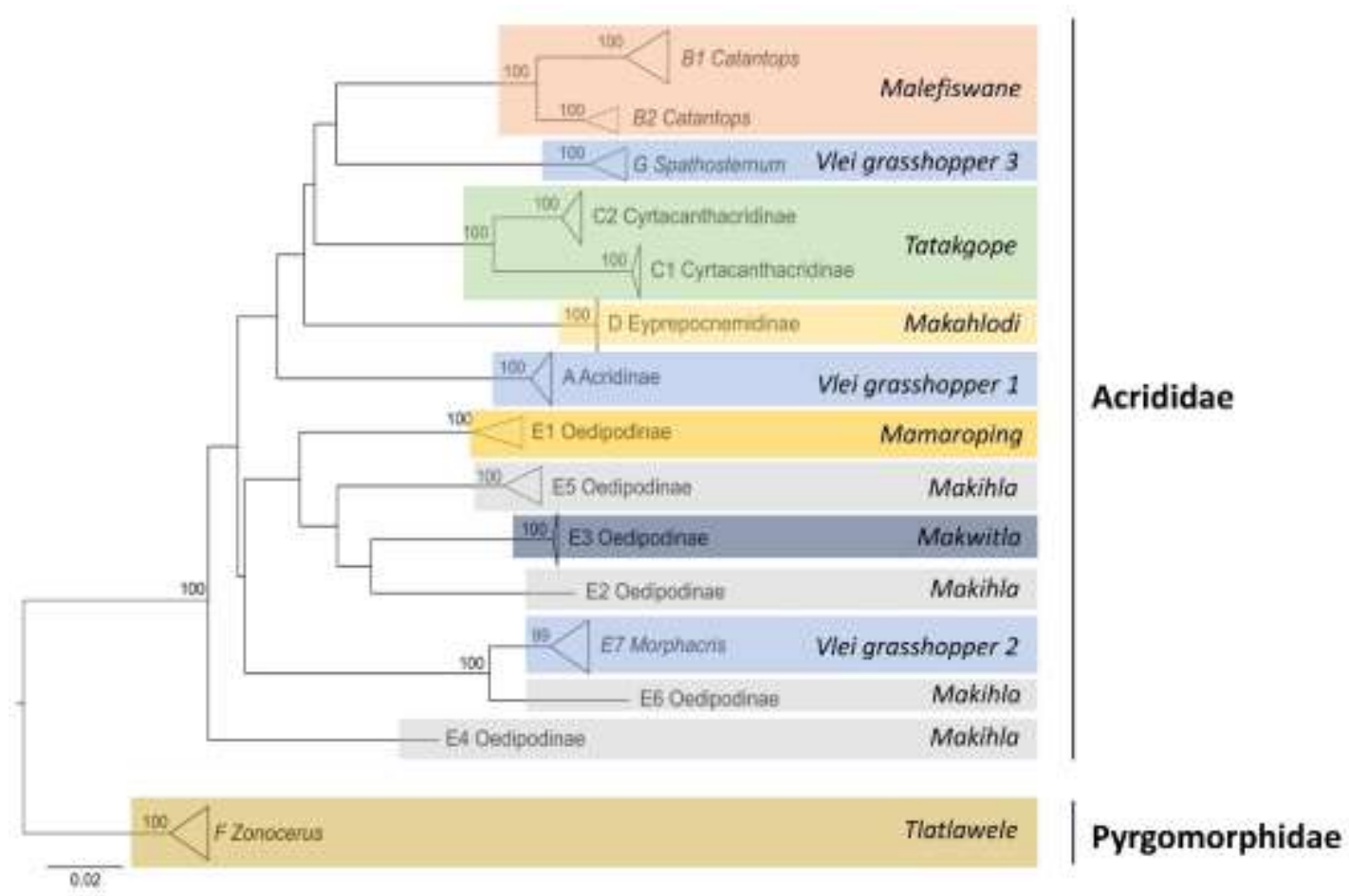

Figure 1. NJ tree of edible grasshoppers found in South Africa. A to G represent 15 different genetic groups, and colour blocks represent seven ethnospecies and Vlei grasshoppers utilized and identified in Limpopo by the local communities.

\subsubsection{Ethnospecies in Acrididae: Cyrtacanthacridinae}

Tatakgope was divided into two genetic groups. $\mathrm{C} 1(\max \mathrm{p}$-distance $=0.31 \%) \mathrm{most}$ likely represents a species of Acanthacris, and C2 (max p-distance $=0.63 \%)$ a species of Cyrtacanthacris (Figure 2). Acanthacris ruficornis was non-monophyletic. 


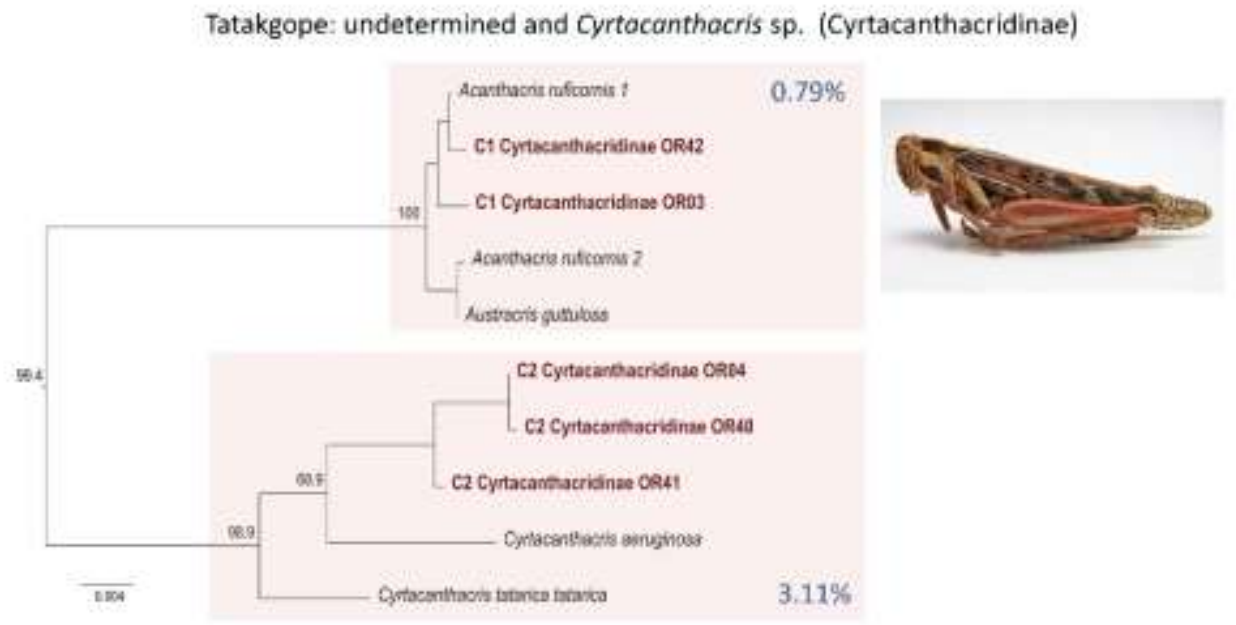

Figure 2. NJ tree of the ethnospecies Tatakgope and the most closely related sequences available on BOLD Systems.

\section{3..2.2. Ethnospecies in Acrididae: Eyprepocnemidinae}

Makahlodi formed genetic group D (max p-distance of $0.00 \%$ ), most closely related to one Heteractis sp. and one Cataloipus sp., but as Heteracris was non-monophyletic, Makahlodi could not be identified to the genus level (Figure 3).

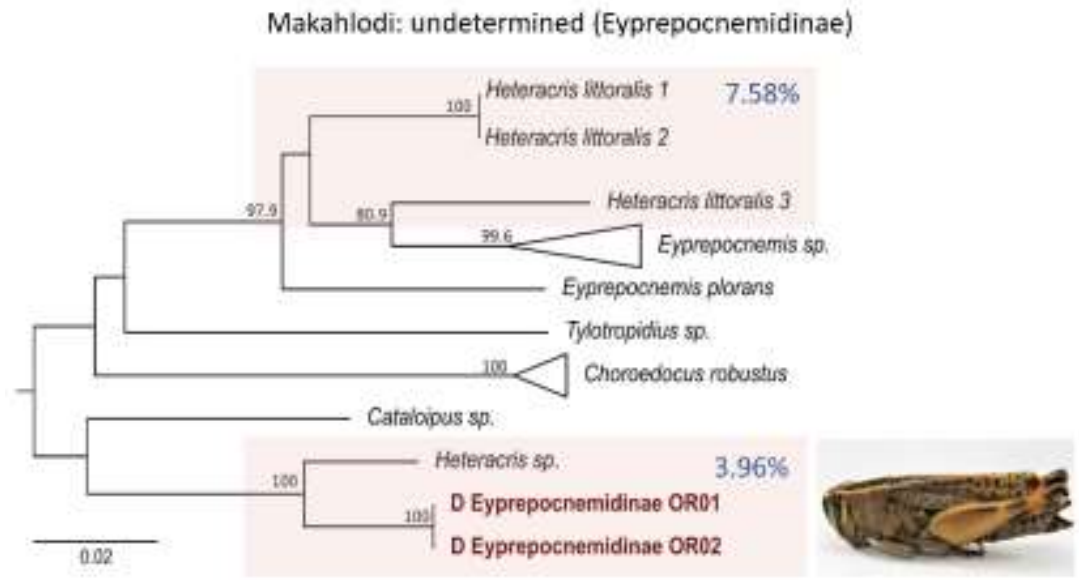

Figure 3. NJ tree of the ethnospecies Makahlodi and the most closely related sequences available on BOLD Systems.

\section{3..2.3. Ethnospecies in Acrididae: Oedipodinae}

Mamaroping formed genetic group E1 (max p-distance $=2.85 \%$ ). The closest relative was Morphacris fasciata with a divergence of 3.33\%, indicating that Mamaroping is in the genus Morphacris but it could represent a distinct species from M. fasciata (Figure 4). 


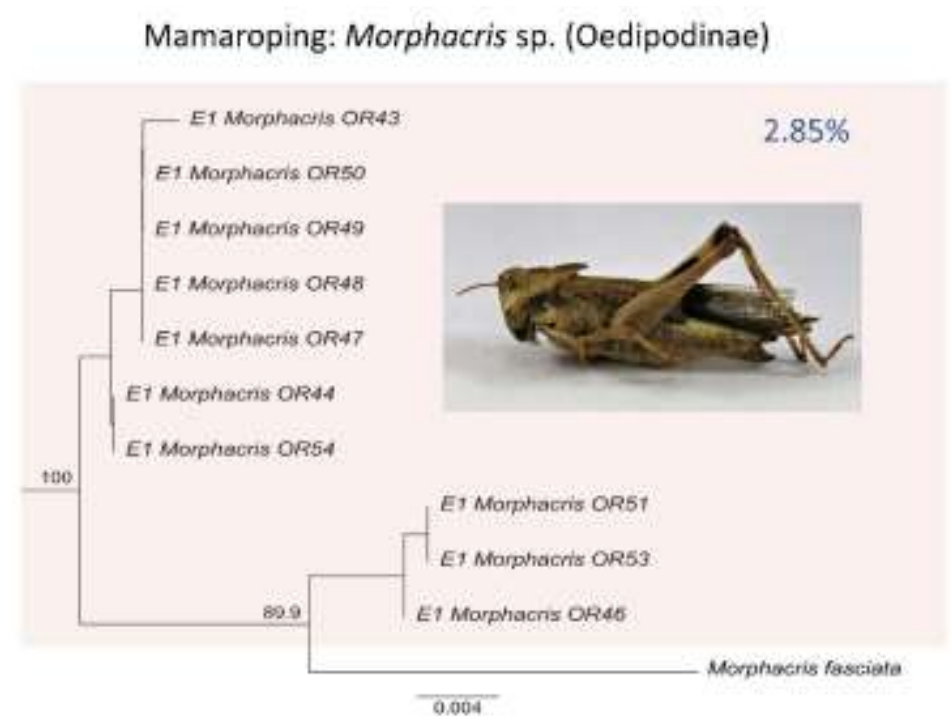

Figure 4. NJ tree of the ethnospecies Mamaroping and the most closely related sequences available on BOLD Systems.

Makwitla formed genetic group E3 (max p-distance $=0.17 \%$ ), which was a sister clade to Gastrimargus africanus sulphureus, G. africanus africanus and G. marmoratus (Figure 5). Makwitla could be a species of Gastrimargus, but a definite conclusion was not possible because Gastrimargus was non-monophyletic.

Makihla was the most genetically diverse ethnospecies with four genetic groups (E2, E4, E5 and E6), all closely related to sequences of Oedaleus but limited data did not allow for reliable diagnostic (Figure 5).

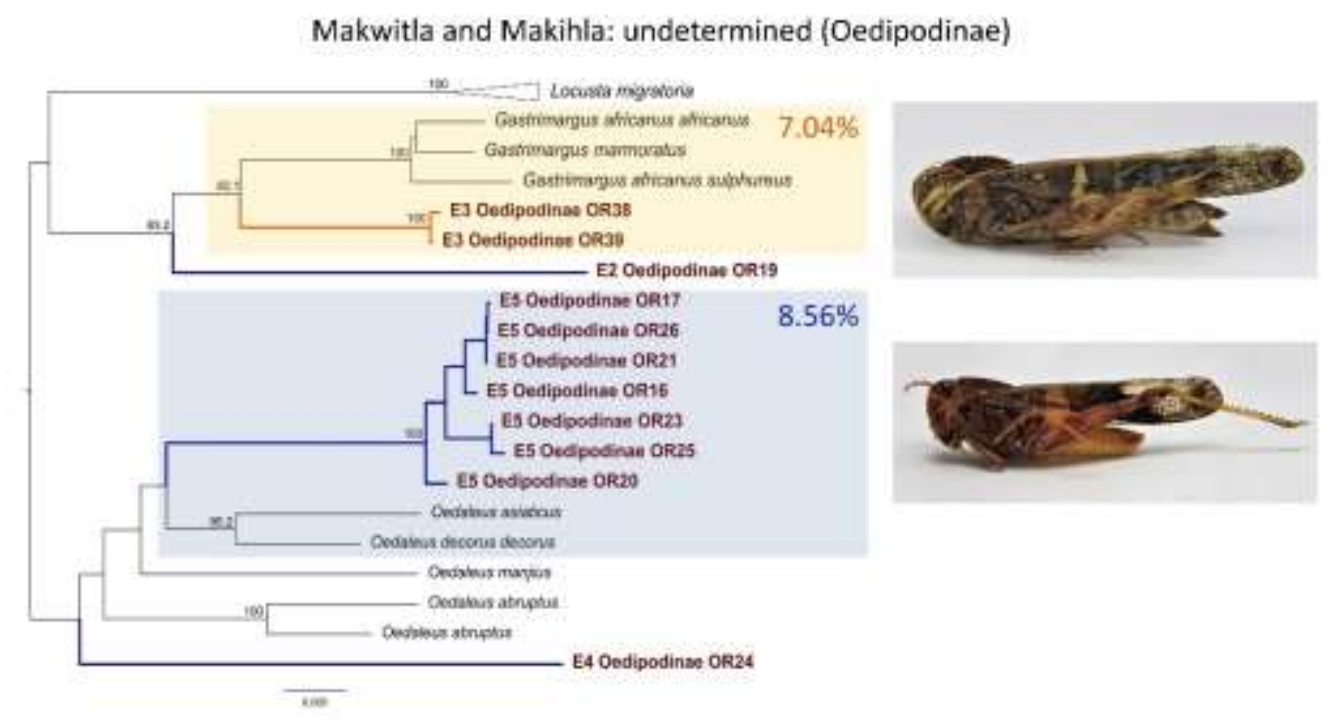

Figure 5. NJ tree of the ethnospecies Makwitla and Makhila and the most closely related sequences available on BOLD Systems.

\subsubsection{Ethnospecies in Acrididae: Catantopinae}

Malefiswane was divided into two genetic groups. B1 (max p-distances 1.37\%) did not cluster with any of the publicly available sequences. B2 clustered with one Catantops sp. sequence with max p-distance of $1.97 \%$. However, the genus Catantops was non-monophyletic (Figure 6). 


\section{Malefiswane: undetermined (Catantopinae)}
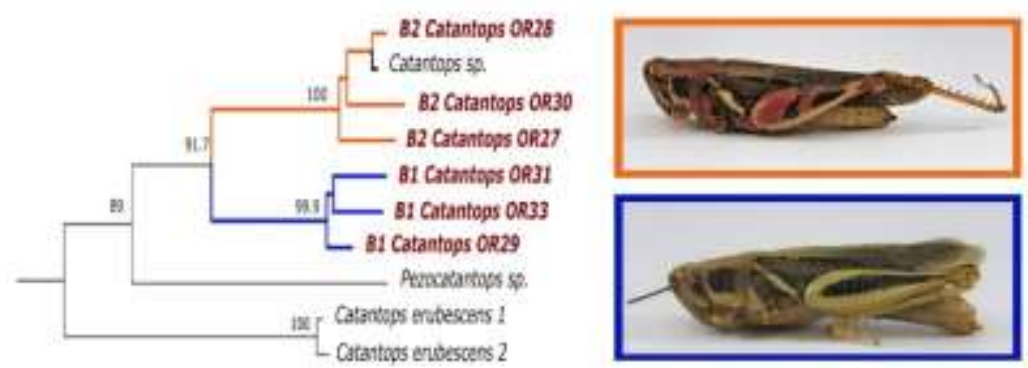

$\overline{0.03}$

Figure 6. NJ tree of the ethnospecies Malefiswane showing the most closely related sequences available on BOLD Systems.

\subsubsection{Vlei grasshoppers}

Vlei grasshoppers are not an ethnospecies per se but a genetic designation of some edible grasshoppers ocasionally consumed. The specimens identified by the communities fell into genetic groups A, E7 and G in the family Acrididae. Vlei grasshoppers of genetic group A (Acridinae) clustered with one Duronia chloronota sequence with a max p-distance of $2.06 \%$ but D. chloronota was non-monophyletic (Figure 7). Vlei grasshoppers in genetic group E7 (Oedipodinae) grouped with Aiolopus thalassinus with max p-distance of $1.79 \%$, and the genus Aiolopus was monophyletic (Figure 8). Vlei grasshoppers in genetic group G (Spathosterninae) formed a cluster with Spathosternum nigrotaeniatum with max p-distance of $2.23 \%$, and the genus Spathosternum was monophyletic (Figure 9).

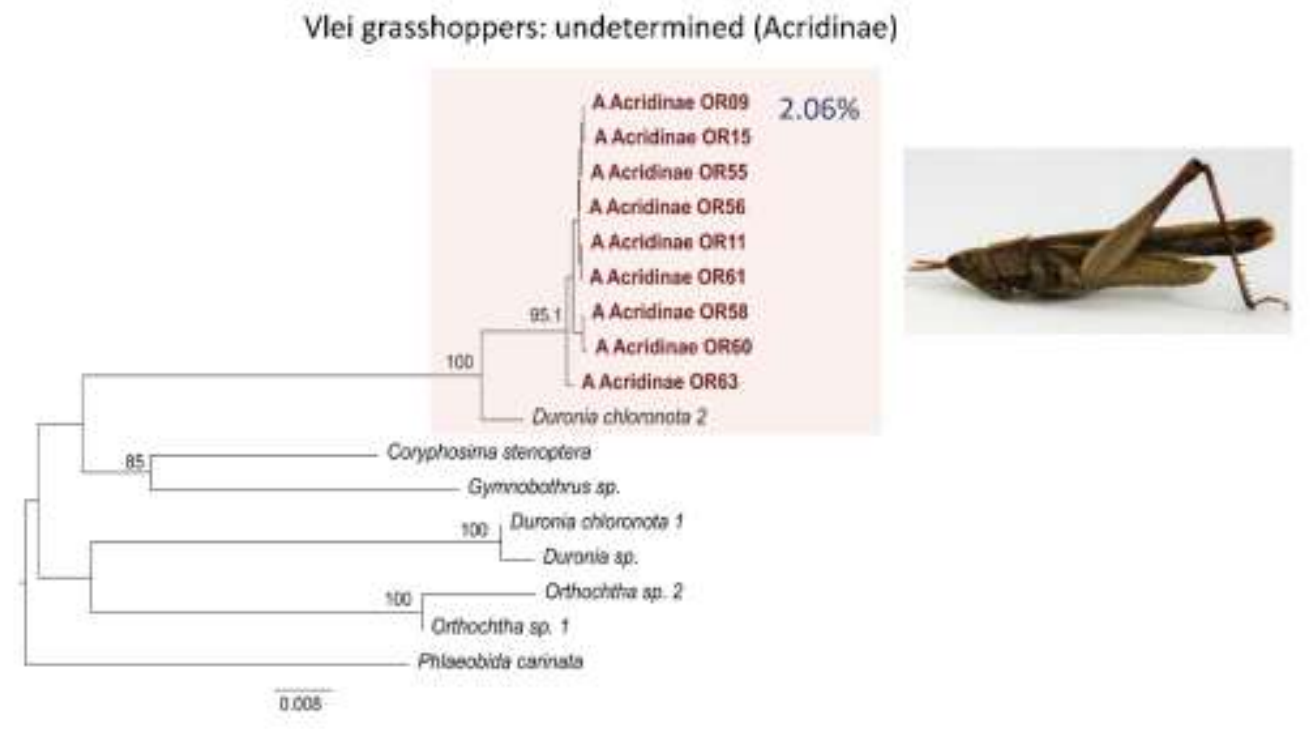

Figure 7. NJ tree of Vlei grasshoppers in Acridinae with the most closely related sequences available on BOLD Systems. 
Vlei grasshoppers: Aiolopus sp. (Oedipodinae)

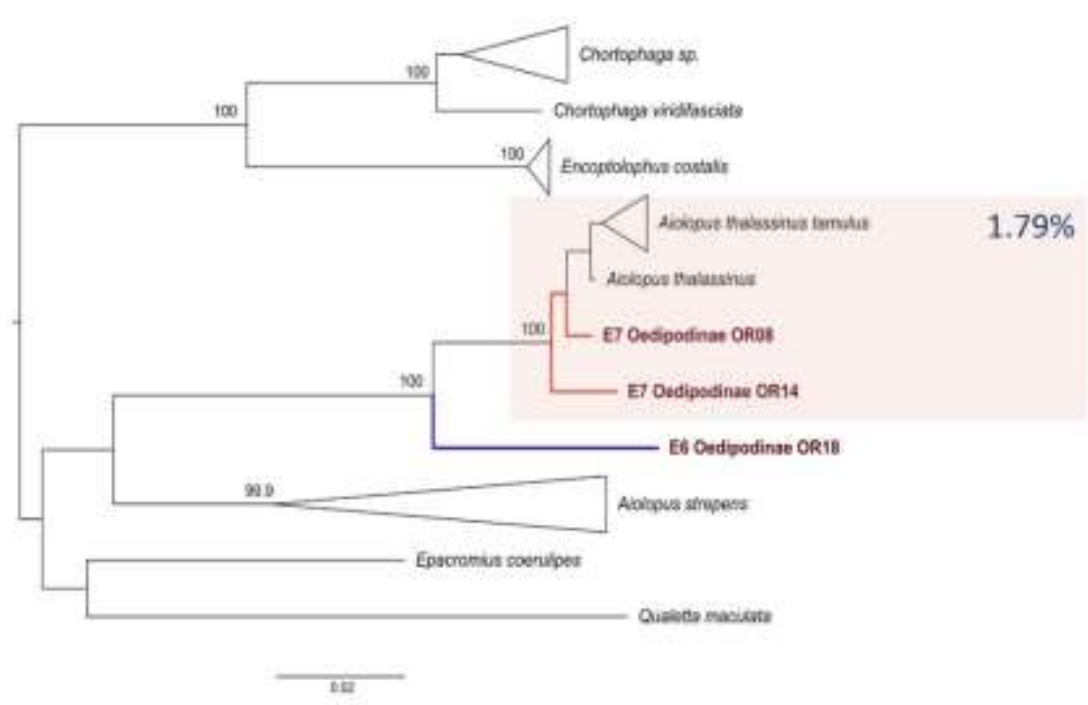

Figure 8. NJ tree of Vlei grasshoppers in Oedipodinae with the most closely related sequences available on BOLD Systems.

Vlei grasshoppers: Spothosternum nigrotaeniatum (Spathosterninae)
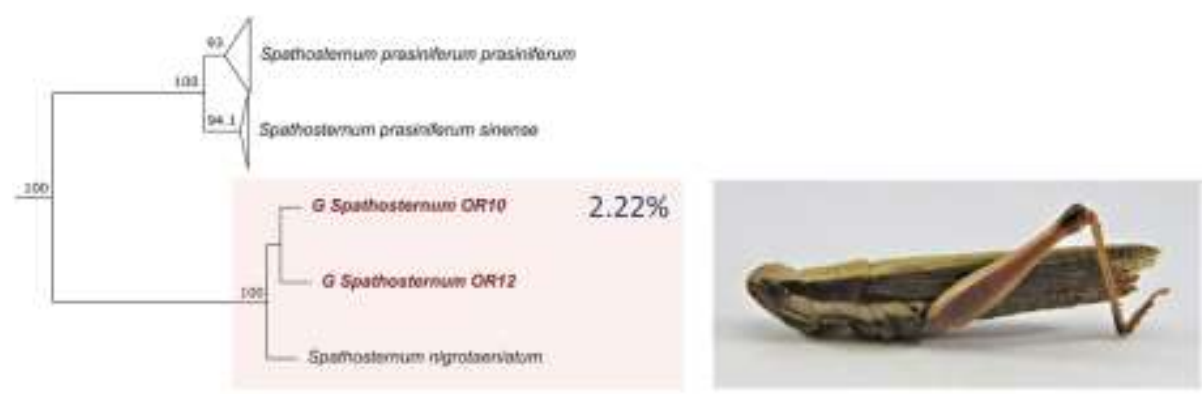

Figure 9. NJ tree of Vlei grasshoppers in Spathosterninae with the most closely related sequences available on BOLD Systems.

\subsubsection{Ethnospecies in Pyrgomorphidae}

Tlatlawele formed genetic group $\mathrm{F}$ with max p-distance of $0.18 \%$, including one Zonocerus elegans sequence available on GenBank, and sister to Zonocerus variegatus (Figure 10). The sequence data available on BOLD Systems indicates that Tatlawele is Zonocerus elegans. 


\section{Tlatlawele: Zonocerus elegans (Pyrgomorphidae: Pyrgomorphinae)}
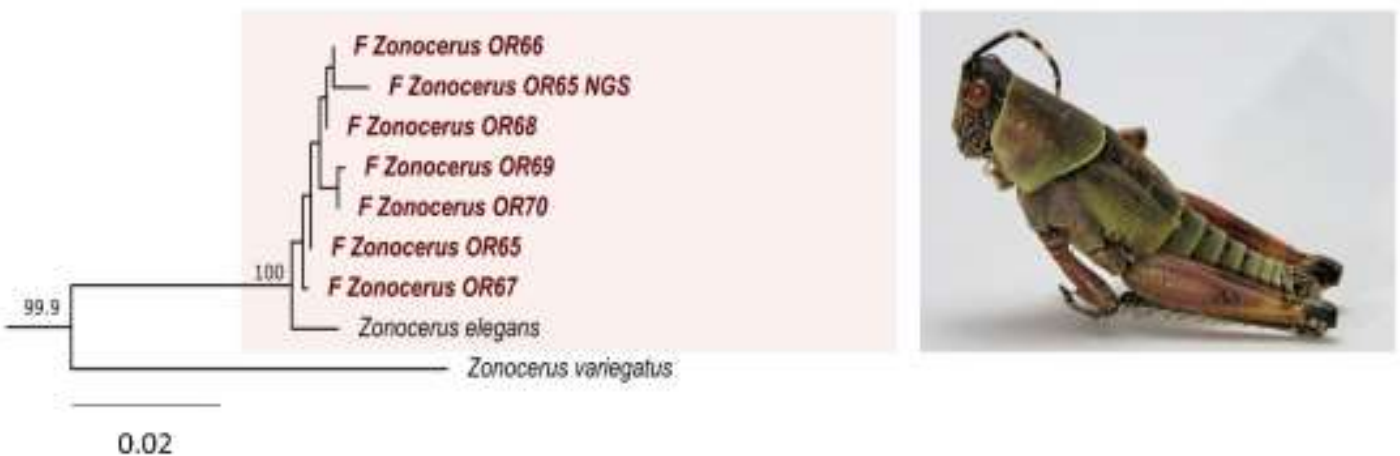

Figure 10. NJ tree of the ethnospecies Tlatlawele with the most closely related sequences available on BOLD Systems.

\section{Conclusion}

The ethnospecies, genetic groups and taxonomic groups of edible insects found in South Africa only overlap partially. The eight ethnospecies and Vlei grasshoppers fall into 15 genetic groups potentially representing distinct species mostly in Acrididae, and only one in Pyrgomorphidae. Only three (Z. elegans, M. fasciata and S. nigrotaeniatum) could be identified to the species level by queries against GenBank and BOLD Systems due to taxonomic inconsistencies, high level of non-monophyly, potential misidentifications and limited data. One ethnospecies (Catantops) was identified to the genus level, and all others were only identified to the subfamily level. Catantops, Z. elegans and M. fasciata are included in the world list of edible insects [6], but M. fasciata is reported here as an edible species in South Africa for the first time, and S. nigrotaeniatum is reported as edible for this first time.

DNA barcoding in Orthoptera is challenging to due widesread presence of NUMTs, and the standard COI region may not offer adequate resolution for species identification.

Supplementary Materials: No supplementary material is included in this paper.

Author Contributions: BVA and BE conceptualized the study. MH performed laboratory work and data analyses. All authors contributed to the writing of the manuscript and approved the final version for publication.

Funding: This research was funded by the Foundational Biodiversity Information Programme (FBIP), grant number 120365 .

Informed Consent Statement: Not applicable.

Data Availability Statement: The sequences generated in this study are not yet publicly available.

Acknowledgments: The authors are grateful to Martin Boima, Nancy Boima, Edmond Ndlovu and Jack Mahlatji for assistance with specimen collection and identification of ethnospecies.

Conflicts of Interest: The authors declare no conflict of interest. 


\section{References}

1. Hanboonsong, Y.; Jamjanya, T. and Durst, P.B. Six-legged livestock: edible insect farming, collecting and marketing in Thailand. Food and Agriculture Organization of the United Nations, Regional Office for Asia and the Pacific: Bangkok, Thailand, 2013; pp. 8-48.

2. Hlongwane, Z.T.; Slotow, R. and Munyai, T.C. Indigenous knowledge about consumption of edible insects in South Africa, Insects 2021 12, 22.

3. Egan, B. Culturally and economically significant insects in the Blouberg Region, Limpopo Province, South Africa. PhD, University of Limpopo, Polokwane, South Africa, 2013.

4. Ribeiro, L. Mitochondrial pseudogenes in insect DNA barcoding: differing points of view on the same issue, Biota Neotropica 2012 12, 301-308.

5. Pereira, R. J.; Ruiz-Ruano, F.J.; Thomas, C.J.E.; Pérez-Ruiz, M.; Jiménez-Bartolomé, M.; Liu, S.; de la Torre, J.; Bella, J.L. Mind the NUMT: Finding informative mitochondrial markers in a giant grasshopper genome. Zool Syst Evol Res. 2021 59, $635-645$.

6. Jongema, Y. Worldwide list of recorded edible insects. Department of Entomology of Wageningen University, The Netherlands, Wageningen. 2017; pp. 17-22. 